\title{
Quantitative Determination of Photosensitivity Proximity Effects in Multi Exposure Direct UV Writing for High Density Integrated Optics
}

\author{
F.R. Mahamd Adikan, J.C. Gates, C.B.E. Gawith and P.G.R. Smith \\ Optoelectronics Research Centre (ORC), University of Southampton, SO17 1BJ United Kingdom
}

UV direct writing is used to write planar channel waveguide gratings and simultaneously investigate photosensitivity proximity effects. Increases are seen up to $9 \mu \mathrm{m}$ away from the initial exposure with maximum effective index increase of $8.3 \times 10^{-4}$.

Price competition and the need for miniaturisation provide powerful incentives for engineers to seek higher device densities in integrated optics. One of the more recently developed techniques known to be capable of fabricating high density integrated optical devices is direct UV writing [1]. This technique has in the past demonstrated successful fabrication of directional power splitters, Bragg gratings and small angle X-couplers. A major advantage of direct UV writing over conventional photolithography and etching techniques is the ability to tailor the refractive index of a given wave guiding structure by exposing the same region with UV light repeatedly, thus improving the overall device performance as reported in [2]. However, little is known about the photosensitivity behaviour of regions within the immediate vicinity of an earlier UV exposed area, also known as the proximity effect. This effect is critical when fabricating high density devices via direct UV writing due to the reduced spacing between two independent structures. Here we present an approach for quantitative characterisation of this effect. Determination of photosensitivity levels at a fixed spacing from an initially exposed region is obtained by defining a set of Bragg gratings with different Bragg wavelengths, and subsequently using the effective index information to deduce the UV induced index increase.

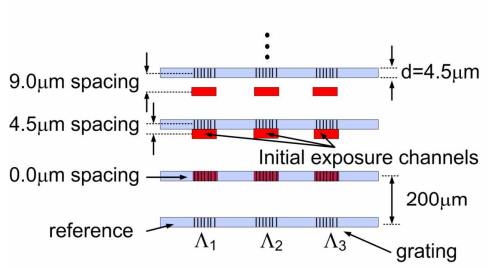

(a)

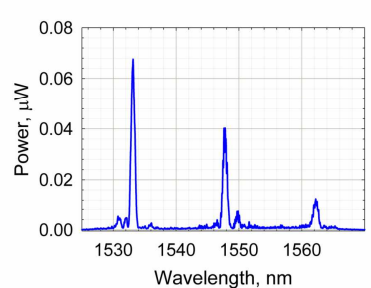

(b)

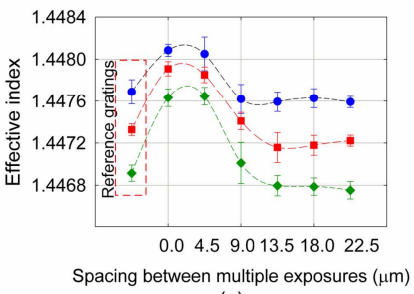

(c)

Fig.1 (a) UV-written Bragg gratings and earlier defined channels for proximity effect characterisation (d: waveguide width); (b) Example of the gratings reflection spectra; (c) Effective index at various spacing and fluence values ( $\bullet$ : Fluence, $\left.F=22 \mathrm{kJcm}^{-2}, \square: F=14 \mathrm{kJcm}^{-2}, \nabla: F=9 \mathrm{kJcm}^{-2}\right)$.

For our initial experiments, sets of three 1mm long Bragg gratings with three different periods $\left(\Lambda_{1}=529.3\right.$, $\Lambda_{2}=534.5, \Lambda_{3}=539.6 \mathrm{~nm}$ ) were defined at fixed spacing from a set of earlier defined straight $1 \mathrm{~mm}$ length regions as shown in Fig. 1(a), on $10 \times 20 \mathrm{~mm}^{2}$ hydrogen-loaded 3 layer silica-on-silicon samples with a 5.6 $\mu$ m thick germanium doped core layer. We found that using three gratings allowed us to overcome uncertainties due to coupling between the two adjacent channels as this coupling effect is wavelength dependent. Reflection spectrums of the gratings were then recorded (Fig. 1(b)), allowing calculation of the average effective index of the waveguide, as depicted in Fig. 1(c). Using different fluence levels facilitates the determination of the point where the photosensitivity is fully depleted and from Fig. 1(c), it is evident that this bleaching point is $>22 \mathrm{kJcm}^{-2}$. Fluence is an expression for the energy exposed to the sample during the UV writing process. It can be seen that an increase in index is obtained in regions up to $9 \mu \mathrm{m}$ away, highlighting the proximity effect, and with a maximum increase obtained when the same region is multi-exposed (0 $\mathrm{mm}$ spacing). Effective index increases as large as $8.3 \times 10^{-4}$ (with respect to the reference gratings) were recorded. We believe that the behaviour exhibited is either due to non-localised heating effects or more likely a result of diffusion of hydroxyl $(\mathrm{OH})$ species resulting in redistribution of oxygen deficient defects, causing an increase in photosensitivity.

Based on these initial results, we will discuss how the proximity effect can be applied to the design of optimised UV written architectures for high density integrated optics. Particular examples will include asymmetric Xcouplers and multi-mode planar Bragg gratings for use in sensing and telecommunication applications.

[1] M. Svalgaard, C. V. Poulsen, A. Bjarklev, and O. Poulsen, "Direct UV writing of buried singlemode channel waveguides in Ge-doped silica films," Electronics Letters, vol. 30, pp. 1401-3, 1994.

[2] F. R. M. Adikan, C. B. E. Gawith, P. G. R. Smith, I. J. G. Sparrow, G. D. Emmerson, C. Riziotis, and H. Ahmad, "Design and demonstration of direct UV-written small angle X-couplers in silica-on-silicon for broadband operation," Applied Optics, vol. 45, pp. 6113-6118, 2006. 